\title{
THE CENTER FOR ASTROPHYSICS REDSHIFT SURVEY
}

\author{
J. Huchra, M. Geller, V. de Lapparent and R. Burg \\ Center for Astrophysics \\ 60 Garden Street \\ Cambridge, MA 02138 USA
}

\begin{abstract}
The first CfA Survey is now over $90 \%$ spectroscopically complete. Over $60 \%$ of all galaxies exhibit detectable emission lines. We have also completed five slices of the Center for Astrophysics (CfA) redshift survey extension. The geometry of the structures in the first slice persists; galaxies are distributed on thin surfaces of "bubble-like" structures. Empty regions or "voids" are common, come in a variety of sizes ranging up to $5,000 \mathrm{~km} \mathrm{~s}^{-1}$, and are underdense by factors of up to 5 w.r.t. the mean. These voids fill $\sim 80 \%$ of the volume of the local universe. Clusters of galaxies lie at the interstices of bubbles; some of the poor Abell clusters do not exist as "fingers" in redshift space. The surfaces are very thin with an average FWHM less than $500 \mathrm{~km} \mathrm{~s}^{-1}$ in redshift space $\left(\sigma \sim 200\right.$ to $\left.250 \mathrm{~km} \mathrm{~s}^{-1}\right)$.
\end{abstract}

\section{INTRODUCTION}

The last decade has seen a renaissance in the observational study of the large scale structure of the universe due to improvements in our ability to measure redshifts of galaxies quickly and accurately. Maps of the redshift space distribution of galaxies and statistical parameters derived from such maps serve both as critical tests of existing cosmological models and as keys to developing new models. In addition, such spectroscopic surveys of nearby galaxies provide necessary baseline samples against which properties of high redshift galaxy samples can be compared to study galactic evolution.

\section{DESCRIPTION OF THE SURVEY}

The first CfA redshift survey was completed in 1981 (Huchra et al. 1983). This 
survey contains all 2400 galaxies with magnitudes brighter than or equal to $\mathrm{m}_{\boldsymbol{z} w}=$ 14.5 and with

or

$$
\delta \geq 0 .^{\circ} 0 \text { and } b^{I I} \geq 40^{\circ}
$$

$$
\delta \geq-2 .^{\circ} 5 \text { and } b^{I I} \leq-30^{\circ} \text {. }
$$

Since its publication, we have classified all of the previously unlcassified galaxies (about 600) from the KPNO glass copies of the Palomar Sky Survey. We have also slowly been obtaining optical spectroscopy for all of the galaxies in that sample where redshifts had previously been obtained only from the literature. We are now over $92 \%$ complete.

In 1984, after several years of concentrating on observations of dynamically interesting systems (eg. Beers et al. 1984, Bothun et al. 1983, Huchra, 1985) we decided to extend the original CfA redshift survey over a similar area of the sky to $\mathrm{m}_{z w}=15.5$. This survey will include all galaxies in a merge of the Zwicky et al. $(1961-1968)$ and Nilson (1973) catalogs between $8^{h}$ and $17^{h}$ in the north galactic cap, and between $20^{h}$ and $4^{h}$ in the south galactic cap. There will be $\sim 15,000$ galaxies in the complete survey; over 8000 redshifts have already been measured.

\section{THE SPECTROSCOPIC SURVEY}

The detailed results of our nearly spectrocopically complete sample are in Burg (1987). Two simple and important results are the relative space densities of galaxies of different morphological type and the frequency of detectable gaseous emission. Both are useful for studying galactic evolution. For example, the combination of the relative space densities with average spectral energy distributions can be used to predict the relative frequency of morphological types either as a function of redshift or of limiting apparent magnitude.

A simple estimate of the absolute space space densities of galaxies can be derived from the V/ $V_{m}$ estimator (Schmidt 1968; Huchra and Sargent 1973). This estimator is known to be biased in the presence of large density inhomogeneities in the sample (see de Lapparent, Geller and Huchra 1988), but the bias will not significantly change the relative space densities of different morphological types in the CfA survey, since all types of galaxies exhibit similar behavior in their $\mathrm{V} / \mathrm{V}_{m}$ distribution (Burg 1987). Figure 1 shows the differential luminosity function (galaxies per magnitude interval per cubic megaparsec) for several Hubble types in the sample. Table 1 gives the approximate Schechter (1976) function parameters derived for each range of types.

The integrated luminosity function we derive is in good agreement with those of Kirshner et al. (1979) and Shanks, et al. (1983). With the exception of the 
TABLE 1

LUMINOSITY FUNCTION PARAMETERS

\begin{tabular}{|c|c|c|c|c|c|}
\hline Type & Range & $\mathbf{N}$ & $\phi^{*}$ & $\mathbf{M}^{*}$ & $\alpha$ \\
\hline E-SO & -7 to -1 & 722 & $4.26 \mathrm{E}-03$ & -19.60 & -1.30 \\
\hline Sab & 0 to 2 & 466 & $4.54 \mathrm{E}-03$ & -19.17 & -1.08 \\
\hline Sbc & 3 to 5 & 877 & $7.50 \mathrm{E}-03$ & -19.29 & -1.08 \\
\hline Scd & 6 to 8 & 200 & $1.92 \mathrm{E}-03$ & -19.06 & -1.56 \\
\hline SmIm & 9 to 11 & 147 & $0.47 \mathrm{E}-03$ & -19.43 & -2.08 \\
\hline All & & 2400 & $15.60 \mathrm{E}-03$ & -19.46 & -1.34 \\
\hline
\end{tabular}

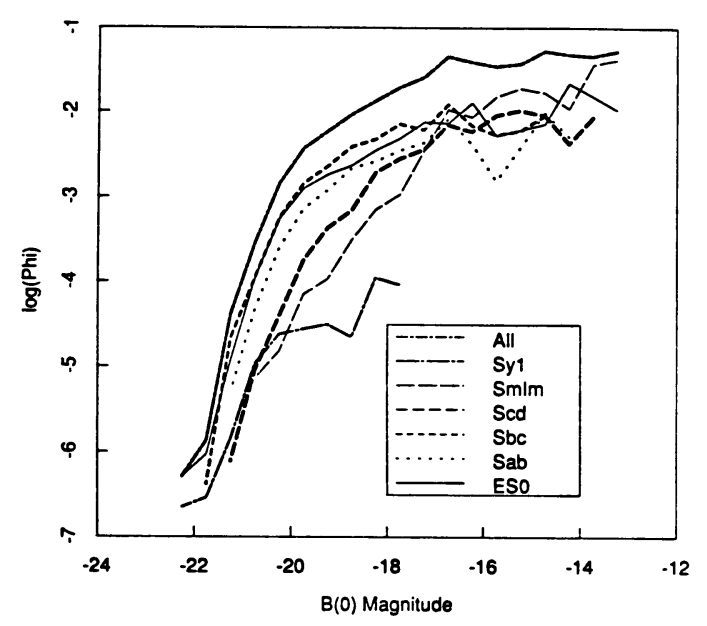

Figure 1. The logarithm of the differential luminosity function, $\phi\left(\# \mathrm{mag}^{-1} \mathrm{Mpc}^{-1}\right.$, by morphological type. "All" refers to the whole sample; the individual distributions for the Hubble types include Seyfert 1 galaxies of those types.

very late type galaxies and the Seyfert galaxies the forms of $\phi$ for the various morphological types are remarkably similar, with $\alpha$ and $\mathrm{M}^{*}$ the same to within the errors. The slope of the luminosity function, $\alpha$, for the Magellanic spirals and irregulars is steeper than that for the other types, while the Seyfert galaxies have a much brighter characteristic magnitude.

The redshift survey spectra are taken with a photon-counting Reticon (Latham 1982 ) on the $1.5-\mathrm{m}$ Tillinghast Reflector at Mt. Hopkins. The spectra typically 
cover $4500-7200 \AA$ at a resolution of $\sim 6 \AA$. The detection limits for emission lines at $\mathrm{H} \alpha$, the O[III] line at $5007 \AA$ or the N[II] line at $6584 \AA$ are typically better than an equivalent width $2.5 \AA$. We can define the fraction of emission line galaxies as the number of galaxies of a given Hubble type relative to the total number that have better than a $5 \sigma$ detection in any of the above lines. That fraction is shown in Figure 2. Over $60 \%$ of all galaxies in a blue magnitude limited sample have detectable emission lines. Because of K-corrections, the fraction of emission line galaxies in an optically selected sample can only rise with redshift.

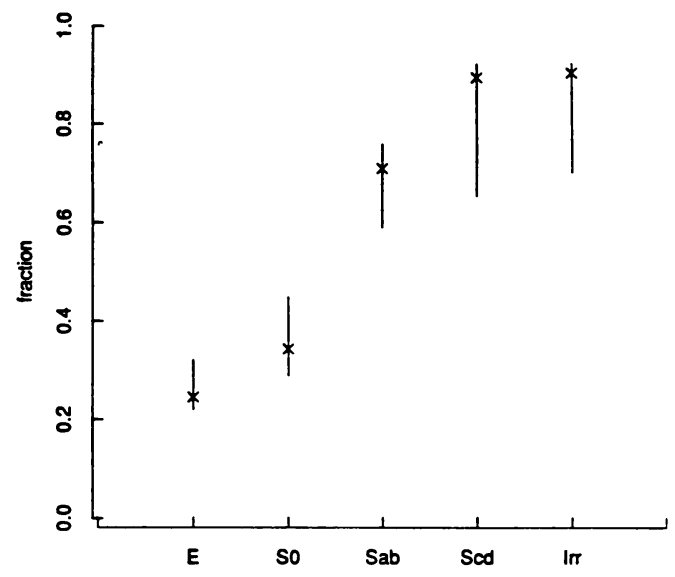

Figure 2. The observed frequency of emission line galaxies versus Hubble type.

\section{THE SLICES}

We have now completed five $6^{\circ}$ slices in the survey extension. The three in the northern hemisphere are contigous. Figure 3 shows the distribution of redshifts for the galaxies in those three strips. To show the structures in more detail, we have plotted all galaxies with redshifts regardless of whether they satisfy the apparent magnitude limit. Figure 4 shows the distribution for the two southern cap slices, which are not contiguous. With the exception of the dense, virialized core of the Coma cluster, the features seen in the four new strips are similar to those seen in the first (de Lapparent, Geller and Huchra 1986).

Statistical analyses of the the data in the first two strips have been done by de Lapparent $(1986,1988)$ and de Lapparent, Geller and Huchra (1988). The spatial two-point correlation function, $\xi(s) \sim\left(s / s_{0}\right)^{\gamma}$, has slope $\gamma \approx 1.6$, and correlation length $s_{0} \approx 7.5 h^{-1} \mathrm{Mpc}$. This length is larger than the estimate of Davis and 

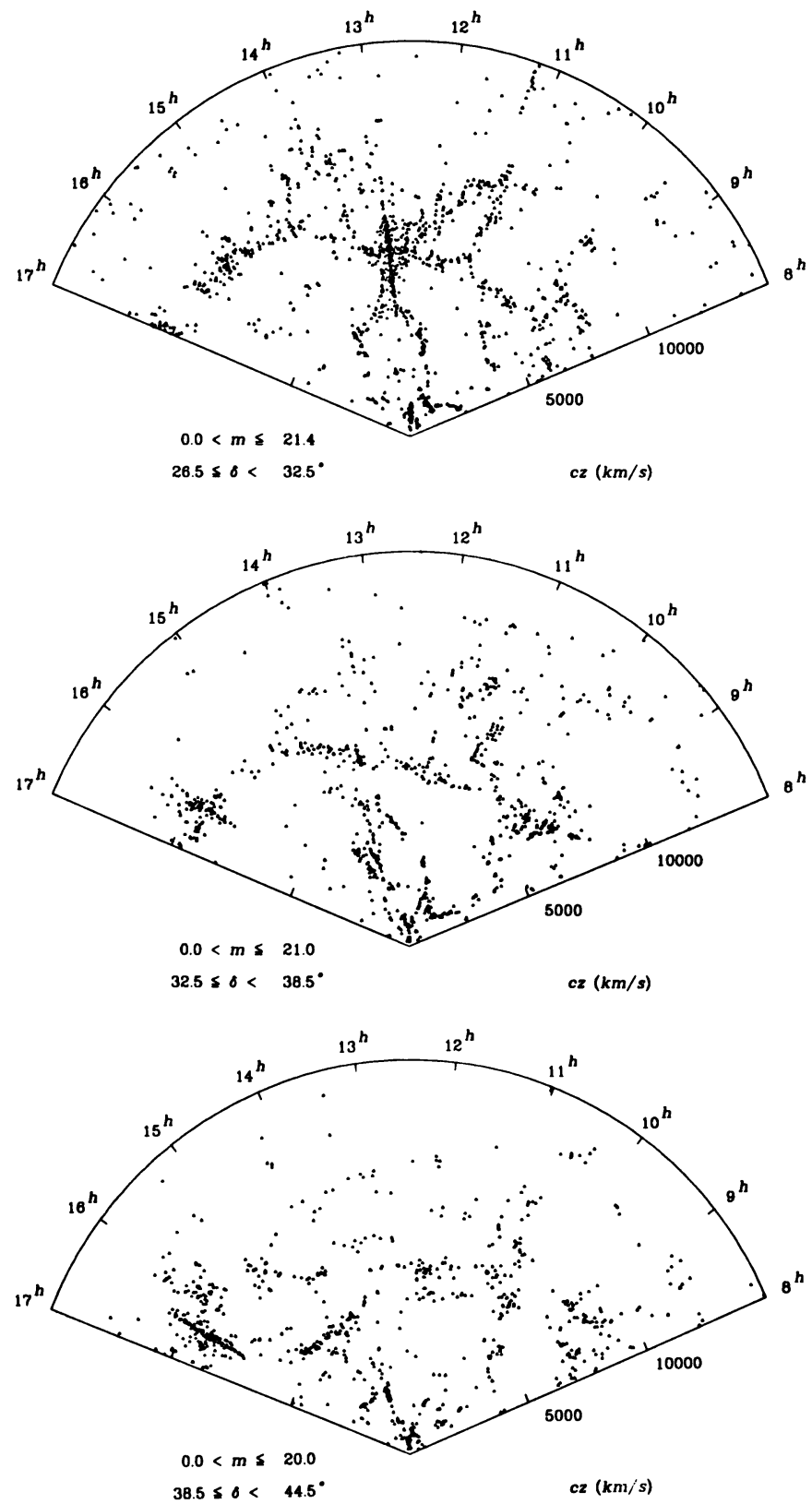

Figure 3a. (top) Observed velocity versus right ascension for all galaxies with known velocities $\leq 15,000 \mathrm{~km} \mathrm{~s}^{\diamond 1}$ in the strip centered at $\delta=29.5^{\circ}$. 2b. (center) The strip centered at $35.5^{\circ}$. 2c. (bottom) The strip centered at $41.5^{\circ}$. Each strip is $6^{\circ}$ thick in declination. 
Peebles (1983). Voids are common in the survey, range in diameter up to the diameter of the Boötes Void (Kirshner et al. 1987), and "fill" slightly less than $80 \%$ of the sample volume. The surfaces surrounding the voids are very thin in redshift space, i.e. have very small perpendicular velocity dispersions. The average FWHM is less than $500 \mathrm{~km} \mathrm{~s}^{-1}$. The voids are underdense relative to the mean galaxy density by factors of up to 5 , and relative to the average density in the filled regions by factors up to 20 .
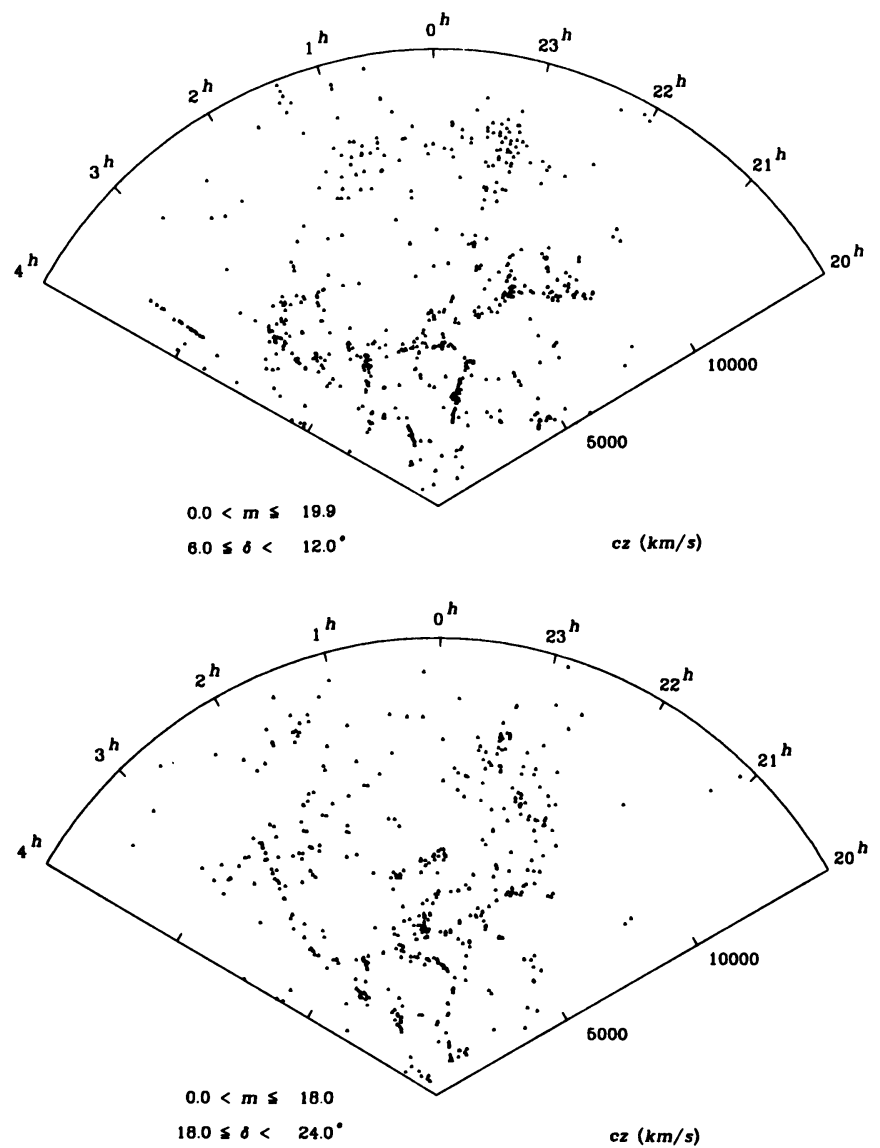

Figure 4. Observed velocity versus right ascension for the two complete strips in the southern galactic cap (a) (top) centered on $+9^{\circ}$, (b) (bottom) centered on $+27^{\circ}$.

We have also compared our redshift maps with nearby clusters of galaxies in 
the Abell (1958) catalog. In the three northern cap strips there are 9 clusters with velocities less than $11,000 \mathrm{~km} \mathrm{~s}^{-1}$ that should appear as "fingers of God" in Figure 3. Only three are easily identifiable, Coma, A2197 and A2199, while the others are only weakly extended in redshift space. There are many structures with greater densities and dispersions which were not classified as clusters by Abell.

\section{THE FUTURE}

One future need is clear from our current surveys. First, all exisiting redshift surveys contain structures as large as could have been seen. The universe is not homogeneous over the scale of our largest surveys. We also will soon exhaust existing catalogs; at the current rate of redshift measurement we will have nearly completed the Zwicky-Nilson merge in five years and will have completed the Abell cluster catalog even before that.

The existing galaxy catalogs are fairly incomplete at their stated limits, and, to make matters worse, the catalogs in the northern and southern hemisphere are not selected in the same way - the Zwicky catalog is magnitude limited, the Nilson catalog is diameter limited, the ESO catalog is diameter limited but on a different scale, and the region in between is covered only by the Vorontsov-Velyaminov et al. (1962-8) catalog which is terribly incomplete (Chapman, Geller and Huchra 1988).

The existing cluster catalogs, Abell and Zwicky, both suffer from a variety of selection effects which significantly limit their usefulness at redshifts greater than 0.1 (eg. Postman, Geller and Huchra 1986, and the results of Struble and Rood 1987, which can also be interpreted as deficiencies in the Abell catalog).

We believe there is a strong case for undertaking a digital sky survey covering both hemispheres which is designed to identify galaxies to 19 th or 20th magnitude and to measure magnitudes for galaxies brighter than 18th magnitude to better than $5 \%$. Such a survey could be done in two colors (both $B$ and $R$ would be useful since most existing catalogs have been compiled in the blue) in $\sim 5$ years.

This research is supported by the Smithsonian Institution and by NASA grant NAGW-201. We would like to thank the CfA redshift survey team, Ed Horine, April Michel, Jim Peters and Susan Tokarz for assistance. Typing services provided by Fang, Inc.

\section{REFERENCES}

Abell, G. O. 1958, Ap. J. Suppl. 3, 211. 
Beers, B., Geller, M. Huchra, J., Latham, D. and Davis, R. 1984, Ap. J. 28333.

Bothun, G., Beers, T., Geller, M. and Huchra, J. 1983, Ap. J. 268, 47.

Chapman, G., Geller, M. and Huchra, J. 1988, A. J. in press.

Burg, R. 1987, Ph. D. Thesis, Massachusetts Institute of Technology.

Davis, M. and Peebles, P. J. E. 1983, Ap. J. 267, 465.

Huchra, J. 1985, in The Virgo Cluster, O. Richter and B. Binggeli, eds. (Garching: ESO), p181.

Huchra, J., Davis, M., Latham, D., and Tonry, J. 1983, Ap. J. Suppl., 52, 89.

Huchra, J. and Sargent, W. L. W. 1973, Ap. J. 186, 433.

Latham, D. 1982, in Instrumentation for Astronomy with Large Optical Telescopes, ed. C. M. Humphries (Dordrecht: Reidel), p. 259.

Kirshner, R.P., Oemler, A. and Schechter, P 1979, A.J., 84, 951. A. J., 88, 1285.

Kirshner, R., Oemler, A., Schechter, P., and Shectman, S. 1987, Ap. J. 314, 493.

de Lapparent, V. 1986, Ph.D. Thesis, Université de Paris VII.

de Lapparent, V. 1988, this conference.

de Lapparent, V., Geller, M.J., and Huchra, J.P. 1986, Ap.J. (Letters), 302, L1.

de Lapparent, V., Geller, M.J., and Huchra, J.P. 1988, Ap. J. in press.

Nilson, P. 1973, Uppsala General Catalogue of Galaxies (Uppsala Astr. Obs. Ann. V), Vol. 1.

Postman, M., Geller, M. and Huchra, J. 1986, A. J. 91, 1267.

Shanks, T., Bean, A.J., Efstathiou, G., Ellis, R.S., Fong, R., and Peterson, B.A. 1983, Ap. J., 274, 529.

Schechter, P.L. 1976, Ap. J., 203, 297.

Schmidt, M. 1968, Ap. J. 151, 393.

Struble, M. and Rood, H. 1987, Ap. J. 323, 468.

Vorontsov-Velyaminov, B. A., Krasnogorska, A. and Arihpova, V. 1962-68 Morphological Catalogue of Galaxies, (Moscow: Moscow State University).

Zwicky, F., Herzog, Wild, P., Karpowicz, M., and Kowal, C. 1961-1968, Catalogue of Galaxies and of Clusters of Galaxies, (Pasadena: California Institute of Technology). 